\title{
In-cruise checkouts of the BepiColombo Laser Altimeter (BELA): Implications for performance and operations at Mercury
}

\author{
Alexander Stark ${ }^{1}$, Hauke Hussmann ${ }^{1}$, Kai Wickhusen ${ }^{1}$, Fabian Lüdicke ${ }^{1}$, Christian Hüttig $^{1}$, Klaus \\ Gwinner $^{1}$, Nicolas Thomas ${ }^{2}$, Luisa M. Lara ${ }^{3}$, and the BELA team \\ ${ }^{1}$ DLR, Department of Planetary Geodesy, Berlin, Germany (hauke.hussmann@dlr.de) \\ ${ }^{2}$ Physikalisches Institut, University of Bern, Switzerland (nicolas.thomas@space.unibe.ch) \\ ${ }^{3}$ Instituto de Astrofísica de Andalucía-CSIC, Aptd. 3004, 18080 Granada, Spain (lara@iaa.es)
}

The BepiColombo Laser Altimeter (BELA) is one of ten payloads on-board the Mercury Planetary Orbiter (MPO) on cruise to Mercury as part of the ESA and JAXA mission BepiColombo. BELA is a single beam laser altimeter operating at $1064 \mathrm{~nm}$. The nominal pulse repetition is $10 \mathrm{~Hz}$, while the pulse energy of each transmitted pulse is $50 \mathrm{~mJ}$. The detector features a silicon avalanche photo diode (APD) with enhanced quantum efficiency for infrared radiation. The detected return pulse signal can be amplified using 16 predefined gain settings within the Analogue Electronics Unit (AEU). Finally, the amplified signal is digitized with a sampling frequency of $80 \mathrm{MHz}$, i.e. in samples of 12.5 ns, and is analysed by an on-board signal processing unit, the Range Finder Module (RFM). Within this pulse processing, nominal values for time of flight are determined for up to four alternative return pulse candidates. Additional parameters such as pulse width and pulse energy are obtained.

Since launch in 2018 the receiver, power converter, and data processing modules of BELA were checked regularly twice a year. The instrument showed excellent performance and all tested parts are operating nominaly. However, the transmitter was not operated because the line-of-sight of the lasers is right in the direction of the BepiColombo transfer module. Hence, the instrument's data acquisition and processing were tested with dark noise measurements.

The expected power of the return pulse from Mercury's surface will vary roughly between 1 and few hundred $\mathrm{nW}$, depending on altitude and surface slope. In order to face this large variability, 16 gain settings with signal amplifications between 4 and $44 \mathrm{~dB}$ will be used for different phases within the orbit. The measurements during the cruise checkouts were used to characterize the noise of the receiver as a function of the commanded gain. By simulating realistic return pulse signals over one revolution about Mercury the commanded gain setting for each spacecraft altitude will be optimized with the ultimate goal of reaching the highest accuracy in the range measurement. Here we will report on the results of the first 5 BELA cruise checkouts, performed between 2019 and June 2021.

BELA is a joint German-Swiss project with the participation of Spain. LML acknowledges financial support from the State Agency for Research of the Spanish MCIU through the "Center of Excellence Severo Ochoa" award to the Instituto de Astrofısica de Andalucia (SEV-2017-0709) and from project PGC2018-099425-B-I00 (MCI/AEI/FEDER, UE). 